\title{
Development and validation of the Thai version of the 4 'A's Test for delirium screening in hospitalized elderly patients with acute medical illnesses
}

This article was published in the following Dove Press journal:

Neuropsychiatric Disease and Treatment

24 February 2016

Number of times this article has been viewed

\author{
Sanchai Kuladee \\ Thanavadee Prachason \\ Department of Psychiatry, Faculty \\ of Medicine, Ramathibodi Hospital, \\ Mahidol University, Bangkok, Thailand
}

Background: The English version of the 4 'A's Test (4AT) is a rapid screening tool for delirium with a high sensitivity and specificity among hospitalized elderly patients.

Objective: To develop the Thai version of the 4AT (4AT-T) and assess its validity.

Subjects and setting: A total of 97 elderly patients aged 60 years or above who were admitted to the general medical wards were included.

Methods: Both authors independently translated the English version of the 4AT into Thai and thereafter developed a single reconciled forward translation by consensus. Back translation was performed by a bilingual native English speaker and it was then reviewed to ensure its agreement with the original one. After 24 hours of admission, subjects were enrolled and clinical data collected. Definite diagnosis of delirium was made by a psychiatrist using the Diagnostic and Statistical Manual of Mental Disorders, 4th edition, Text-Revision criteria and the 6-item Thai Delirium Rating Scale; the 4AT was then administered to participants by nurses within 30 minutes. A 4 AT score $\geq 4$ was considered positive for delirium screening. The optimal cut-off point of the 4AT-T was identified by Youden's index.

Results: In all, 24 out of 97 participants met the Diagnostic and Statistical Manual of Mental Disorders, 4th edition, Text-Revision criteria for delirium. At a cut-off score of 4 or greater, the 4AT-T exhibited satisfactory diagnostic performance with a sensitivity of $83.3 \%(95 \%$ confidence interval (CI): $62.6 \%-95.3 \%$ ) and specificity of $86.3 \%$ (95\% CI: $76.3 \%-93.2 \%)$. The area under the receiver operating characteristic curve was 0.92 . The specified score provided maximal Youden's index, suggesting an optimal criterion value for delirium screening.

Conclusion: The 4AT-T is a valid delirium-screening instrument for hospitalized elderly patients with acute medical illnesses.

Keywords: Thai, delirium, screening tool, delirium screening, acute medical illnesses

\section{Introduction}

Delirium is a neurocognitive disorder usually caused by multiple medical conditions that result in generalized brain dysfunction. The essential features include disturbance in attention and other cognitive functions, disorientation to the environment, and fluctuation in severity during the course of the day. ${ }^{1,2}$ The condition is common, especially among older people, and associated with high mortality rates ranging from $22 \%$ to $76 \%$ among hospitalized patients. ${ }^{3}$ Moreover, delirium significantly increases functional disability, costs of care, length of stay, rates of institutionalization, and rates of nursing home placement. ${ }^{2,4,5}$

In general, delirium can be resolved after the underlying etiological factors are promptly corrected, ${ }^{1,6}$ however, the condition is frequently underrecognized, ${ }^{5,7}$ resulting in a delay for appropriate management and probably leading to persistence
Correspondence: Sanchai Kuladee Department of Psychiatry, Faculty of Medicine, Ramathibodi Hospital, Mahidol University, 270 Rama VI Rd, Phayathai, Ratchathewee, Bangkok, Thailand 10400 Tel +6622011929

Fax +66 23547299

Email sanchai.kul@mahidol.edu (c) (1) (-) 2016 Kuladee and Prachason. This work is published and licensed by Dove Medical Press Limited. The full terms of this license are available at https://www.dovepress.com/terms.php
and incorporate the Creative Commons Attribution - Non Commercial (unported, v3.0) License (http://creativecommons.org/licenses/by-nc/3.0/). By accessing the work you

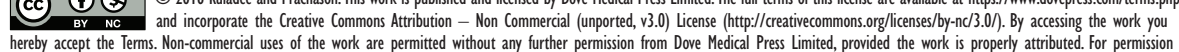
for commercial use of this work, please see paragraphs 4.2 and 5 of our Terms (https://www.dovepress.com/terms.php).
forition 
of the symptoms. ${ }^{6,8}$ A recent systematic review reveals that the rates of persistent delirium among hospitalized elderly patients were indeed as high as $44.7 \%$ at discharge from hospital and 21\% 6 months later. ${ }^{8}$ Delayed recognition and persistence of delirium also appear to be associated with worse outcomes and higher mortality rate in elderly patients. ${ }^{8-10}$ Hence, routine screening for delirium is a key strategy for early detection of the condition and avoiding delayed management. ${ }^{4,6,10,11}$

A large number of instruments for screening, diagnosis, and assessment of delirium have been developed over the past decades. ${ }^{12-14}$ Among these, the Confusion Assessment Method is one of the most extensively used tools for delirium screening according to its high sensitivity and specificity (95\% and $89 \%$, respectively) as well as its brevity in administration. ${ }^{10,11,15,16}$ However, optimal training is required to achieve such high accuracy. To improve practicality of delirium screening, the 4 'A's Test (4AT; www.the4at.com) has been developed as a rapid assessment test for delirium, which requires no specific training. Its satisfactory diagnostic test accuracy has been proved in 234 hospitalized elderly patients evaluated by a geriatrician without prior specific training. ${ }^{17}$ The $4 \mathrm{AT}$ score ranges from 0 to 12 , with a score of 4 or above indicating delirium. The area under the receiver operating characteristic (ROC) curve for delirium detection was 0.93 , with a sensitivity of $89.7 \%$, specificity of $84.1 \%$, positive likelihood ratio of 5.62, and negative likelihood ratio of $0.12 .{ }^{17}$ As compared to other brief screening tools, the 4AT also showed greatest sensitivity (100\%) with reasonable specificity $(82 \%)$ for delirium detection in an acute stroke unit. ${ }^{18}$

In Thailand, the prevalence of delirium has been reported to be as high as $40.4 \%$ among older patients admitted to general medical wards. ${ }^{19}$ In in-patient settings, nurses are health care personnel holding great opportunities to detect subtle changes in mental status of patients. Nevertheless, underrecognition of delirium frequently occurs, ${ }^{20}$ which could be partly attributed to excessive workloads of attending staff. ${ }^{21}$ A study of 5,247 nurses who provided direct care for patients in 39 public hospitals in Thailand found that each nurse was responsible for $7-13$ patients. ${ }^{22}$ To improve detection of delirium, a simple but accurate screening instrument is required particularly in clinical settings with a disproportionately large number of patients, such as in our country. The objective of this study was to evaluate the validity and reliability of the Thai version of the 4AT (4AT-T) as a screening instrument for delirium in general medical settings.

\section{Methods}

We applied the reporting standards for studies of diagnostic test accuracy in dementia in our study. ${ }^{23}$ This study was approved by the Ethics Committee on Human Experimentation of the Faculty of Medicine, Ramathibodi Hospital, Mahidol University, Bangkok, Thailand. Written informed consent was obtained either directly from the participant or from his/her legally authorized representative. This study project continued from April 2014 to September 2015.

\section{Study design and participants}

This study was a cross-sectional study conducted in Ramathibodi Hospital, which is a tertiary hospital with 1,300 beds located in the center of Bangkok, Thailand. We defined "elderly" as an age of 60 years or above according to the National Statistical Office of Thailand. The elderly patients who were admitted to the general medical wards were recruited from December 2014 to February 2015. The medical wards provide care for adults of all ages who were admitted with acute medical conditions, while each staff nurse usually takes care of six to eight patients on an average. The service includes patients transferred from intensive care and intermediate units when critical medical conditions had subsided, and also those transferred from secondary health care centers due to complex medical conditions. We excluded patients with conditions that prohibit adequate communication, including severe hearing impairment, inability to speak, intubation, and coma.

\section{Measures}

\section{Development of the 4AT-T}

After obtaining permission from the copyright holder, the English 4AT version 1.1 was translated into Thai by one psychiatrist (SK) and one psychiatry resident with $\mathrm{PhD}$ (TP). These forward translations were carried out independently, and thereafter these were reconciled into a consensus version. Then, the final Thai version was delivered to a professional translator (IH), a bilingual native English speaker fluent in Thai language for back translation without prior knowledge of the original version. Finally, SK and TP reviewed the back-translated version to ensure concordance between the translated and the original versions.

The 4AT-T consists of four items. First, the alertness item assesses the altered level of alertness. ${ }^{24}$ Second, the 4-item Abbreviated Mental Test (AMT4) ${ }^{25}$ assesses patients' cognition by asking about their age, date of birth, the present place, and the current year. Third, the attention 
item tests patients' attention using the months backwards test. ${ }^{26}$ Lastly, the acute change or fluctuating course item evaluates the onset and course of the altered mental status. The total score ranges from 0 to 12 which is the same as the original version.

\section{6-item TDRS}

The 6-item Thai Delirium Rating Scale (TDRS) is an instrument used for diagnosing and severity grading of delirium. ${ }^{27}$ The evaluated items include temporal onset of symptoms, psychomotor behavior, cognitive status, physical disorder, sleep-wake cycle disturbance, and variability of symptoms. The total score of TDRS ranges from 0 to 20 and positively correlates with the severity of delirium symptoms. ${ }^{27}$ At a cut-off score of 10 or above, the tool showed excellent sensitivity and specificity for the diagnosis of delirium $(97.5 \%$ and $97.8 \%$, respectively) as well as good internal consistency $($ Cronbach's alpha $=0.89) .{ }^{27}$

\section{Procedure}

Demographic and clinical characteristics were derived from a direct interview and clinical records. Charlson comorbidity index, ranging from 0 to 11 , was calculated to determine the seriousness of comorbid diseases of each participant. ${ }^{28}$ After being admitted to the wards for at least 24 hours, each participant was interviewed and assessed by one of the two experienced psychiatrists blinded to 4AT scores; definite diagnosis of delirium was made in accordance with the Diagnostic and Statistical Manual of Mental Disorders, 4th edition, Text-Revision (DSM-IV-TR) criteria. ${ }^{29}$ Additionally, the 6-item TDRS was used to confirm the diagnosis and determine the severity of the symptoms. To be included in delirium group, the DSM-IV-TR diagnosis by the psychiatrist must be delirium and the total score of TDRS must be 10 or above. To evaluate inter-rater reliability, five participants were co-rated with the TDRS and were diagnosed independently by both psychiatrists. All psychiatrists who participated in this study were certified by the Thai Board of Psychiatry and had experience in taking care of patients with delirium.

Within 30 minutes of the evaluation by the psychiatrists, the 4AT-T was administered to each participant by one of the three nurses who work regularly at the wards with no specific training provided. Scoring of the 4AT-T was performed in a blinded fashion to the diagnosis made by the psychiatrists. The inter-rater reliability of the 4AT-T was established in ten participants using a similar process applied to the 6-item TDRS. After completing the evaluation, all the three nurses were interviewed briefly for some feedback about the usage of this new assessment tool.

\section{Statistical analyses}

Data analyses were performed using PASW Statistics for Windows, Version 18.0.0 (SPSS Inc., Chicago, IL, USA) and MedCalc for Windows, Version 15.6.1 (MedCalc Software, Ostend, Belgium). Characteristics of patients with and without delirium were compared using chi-square test for categorical variables and Student's $t$-test for continuous variables. The internal consistency of the 4AT-T was measured by Cronbach's alpha coefficient. Correlations between each item and the total scores of the 4AT-T were determined. The inter-rater reliability for the 4AT-T and 6-item TDRS was assessed with intra-class correlation coefficient. The inter-rater agreement for diagnosis of delirium according to DSM-IV-TR criteria was measured by Cronbach's alpha coefficient.

To evaluate validity of the test, sensitivity, specificity, likelihood ratios, and predictive values of the 4AT-T were calculated using the DSM-IV-TR criteria as a reference standard. The area under the ROC curve was determined. The performance of the 4AT-T against the reference standard was also re-evaluated at various cut-off scores. Youden's index was calculated to determine the optimal criterion value of the test.

\section{Results}

From a total of 237 elderly patients aged 60 years or above who were admitted to the general medical wards during a period of 3 months, 97 were included in this study. Among these, 24 patients had delirium according to DSMIV-TR criteria as well as 6-item TDRS. Demographic and clinical characteristics of the study population are shown in Table 1 . The mean age was 73.6 years (standard deviation $(\mathrm{SD})=8.17)$. There were approximately equal proportions of males and females in both groups of patients. About $60 \%$ of participants had 0-6 years of education. No significant difference of mean age, sex, total years of education, and comorbidity index was observed between the delirium and nondelirium groups.

\section{Reliability and item analysis}

Cronbach's alpha for the diagnosis of delirium based on DSM-IV-TR criteria was 0.998 . Intra-class correlation coefficients for the 6-item TDRS and 4AT-T were $0.973(95 \%$ CI: $0.771-0.997 ; P=0.001)$ and 0.998 (95\% CI: 0.994-0.999; $P<0.001)$, respectively. 
Table I Demographic and clinical characteristics of the study population

\begin{tabular}{llll}
\hline & $\begin{array}{l}\text { Delirium } \\
(\mathbf{N}=\mathbf{2 4})\end{array}$ & $\begin{array}{l}\text { Nondelirium } \\
\mathbf{( N = 7 3 )}\end{array}$ & P-value \\
\hline Age in years, mean (SD) & $75.3(8.1)$ & $73.1(8.2)$ & 0.265 \\
Sex, female N (\%) & $12(50)$ & $36(49.3)$ & 0.954 \\
Years of education, N (\%) & & & \\
$\quad$ I2 years & $3(12.5)$ & $14(19.2)$ & 0.812 \\
7-12 years & I (4.2) & $2(2.7)$ & \\
I-6 years & $15(62.5)$ & $39(53.4)$ & \\
0 & $5(20.8)$ & $18(24.7)$ & \\
Presenting symptoms, N (\%) & & & \\
$\quad$ Acute fever & $5(20.8)$ & $9(12.3)$ & 0.012 \\
$\quad$ Dyspnea & $6(25.0)$ & $3 \mid(42.5)$ & \\
$\quad$ Alteration of consciousness & $5(20.8)$ & $2(2.7)$ & \\
$\quad$ Others & $8(33.3)$ & $3 \mid(42.5)$ & \\
CCl median (IQR) & $3(1.25-5)$ & $4(2-5.5)$ & 0.403 \\
\hline
\end{tabular}

Abbreviations: $\mathrm{CCl}$, Charlson comorbidity index; IQR, interquartile range; SD, standard deviation.

Cronbach's alpha for the internal consistency of the 4AT-T was 0.75. Mean (SD) and corrected item-corrected correlation are shown in Table S1.

\section{Validity}

Twenty out of 24 delirious patients were correctly classified as delirious using the 4AT-T. As compared to the reference standard as well as the 6-item TDRS, the 4AT-T falsely detected ten nondelirious subjects but missed detecting four delirious patients (Table 2). Out of ten patients misclassified by the 4AT-T as delirious, five were found to have dementia according to clinical history from reliable informants, and another two were diagnosed to have organic brain syndrome. The instrument, at a cut-off point of 4 or greater, exhibited a sensitivity of $83.3 \%$ (95\% CI: $62.6 \%-95.3 \%$ ), specificity of $86.3 \%$ (95\% CI: $76.3 \%-93.2 \%$ ), positive predictive value of $66.7 \%$ (95\% CI: $47.2 \%-82.7 \%$ ), negative predictive value of $94.0 \%$ (95\% CI: $85.4 \%-98.4 \%$ ), positive likelihood ratio of 6.1 (95\% CI: $3.3-11.1)$, and negative likelihood ratio of 0.2 ( $95 \%$ CI: $0.1-0.5)$. The ROC curve demonstrated efficient performance of the 4AT- T in

Table 2 Diagnostic performance of the 4AT-T compared with the reference standard

\begin{tabular}{lllll}
\hline & \multicolumn{3}{l}{ Reference standard* } \\
\cline { 3 - 5 } & & Delirium & Nondelirium & Total \\
\hline 4AT-T & Delirium (N) & 20 & 10 & 30 \\
& Nondelirium (N) & 4 & 63 & 67 \\
& Total & 24 & 73 & 97 \\
\hline
\end{tabular}

Note: *Reference standard = DSM-IV-TR diagnosis and TDRS score 10 or above. Abbreviations: 4AT-T, Thai version of the 4 'A's Test; DSM-IV-TR, Diagnostic and Statistical Manual of Mental Disorders, 4th edition, Text-Revision; TDRS, Thai Delirium Rating Scale. the detection of delirium (Figure 1). The area under the curve was 0.92 (95\% CI: $0.86-0.97, P<0.001)$, suggesting high accuracy. ${ }^{30}$

When each subtest was focused on, the highest sensitivity was observed for the attention item and the AMT4 $(95.8 \%$ at a score of 1 in both items). The attention test also exhibited high specificity ( $94.5 \%$ at a score of 2 ). The alertness item showed the highest specificity and positive likelihood ratio but the lowest sensitivity as compared to the others. The diagnostic performance of the 4AT-T and each subtest are shown in Table 3.

To determine an optimal cut-off score of the 4AT-T for delirium screening, the diagnostic performance of the test was re-evaluated at various cut-off values. As shown in Table S2, maximal Youden's index was derived at the cutoff point of $>3$, suggesting the most appropriate diagnostic criterion for delirium.

\section{Feedback from assessors}

All participating nurses provided us some positive feedback about the ease of use and practicality of the 4AT-T. As one nurse mentioned: "All questions of the tool are quite simple and easy to understand. I can complete the assessment within five minutes per case." Another comment was "I think it could be included in our routine works and I feel no burden using it."

On the other hand, there was feedback about the difficulty inquiring the date of birth in the AMT4 section: "A few patients gave me the answer of the year of birth in Chinese zodiac, like the Year of Tiger, and then I had to find out more what the right year was."

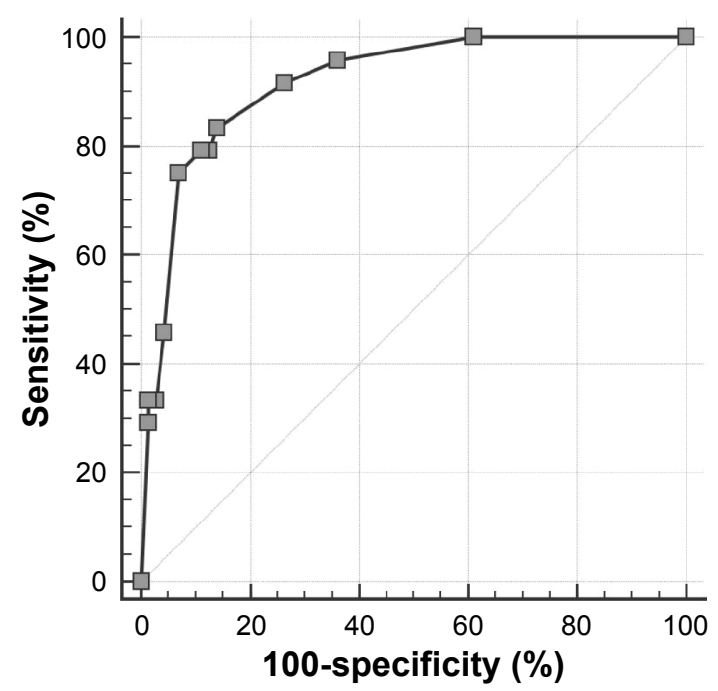

Figure I ROC curve demonstrating the efficient performance of the 4AT-T in the detection of delirium.

Abbreviations: 4AT-T, Thai version of the 4 'A's Test; ROC, receiver operating characteristic. 
Table 3 The performance of the 4AT-T and each subtest in diagnosing delirium

\begin{tabular}{llllllll}
\hline & 4AT total & Alertness & AMT4 & & Attention & & Acute change/fluctuation \\
\hline Cut-off & $\geq 4$ & 4 & 1 & 2 & I & 2 & 4 \\
Sensitivity (\%) & 83.3 & 37.5 & 95.8 & 87.5 & 95.8 & 50.0 & 75.0 \\
Specificity (\%) & 86.3 & 97.3 & 67.1 & 80.8 & 41.1 & 94.5 & 87.7 \\
Positive predictive value (\%) & 66.7 & 81.8 & 48.9 & 60.0 & 34.8 & 75.0 & 66.7 \\
Negative predictive value (\%) & 94.0 & 82.6 & 98.0 & 95.2 & 96.8 & 85.2 & 91.4 \\
Positive likelihood ratio & 6.1 & 13.7 & 2.9 & 4.6 & 1.6 & 9.1 & 6.1 \\
Negative likelihood ratio & 0.2 & 0.6 & 0.1 & 0.2 & 0.1 & 0.5 & 0.3 \\
Area under ROC curve & 0.919 & 0.674 & 0.867 & & 0.806 & & 0.813 \\
P-value & $<0.00 \mathrm{I}$ & $0.0 \mathrm{II}$ & $<0.00 \mathrm{I}$ & & $<0.00 \mathrm{I}$ & & $<0.00 \mathrm{I}$ \\
\hline
\end{tabular}

Abbreviations: 4AT, 4 'A's Test; 4AT-T, Thai version of the 4AT; AMT4, 4-item Abbreviated Mental Test; ROC, receiver operating characteristic.

\section{Discussion}

This study aims to develop and validate a rapid, easy-to-use, screening instrument for delirium in hospitalized elderly patients, a group of the population with a high risk for delirium. The 4AT-T performed well in delirium detection with high sensitivity and specificity. Although the sensitivity of the 4AT-T was less than the original one ${ }^{17}(83.3 \%$ and $89.7 \%$ ), the 4AT-T had a higher specificity $(86.3 \%$ and $84.1 \%)$. Moreover, there is a slight difference in the areas under the ROC curves ( 0.92 and 0.93 ). It also demonstrated a high negative predictive value, which greatly facilitates exclusion of delirium in negative cases. The optimal cut-off value of the test for delirium screening was also consistent with the original version.

Despite its satisfactory performance profile, four out of 24 delirious patients were not detected. These subjects appeared to have TDRS scores ranging from 10 to 13 , implying that the 4AT-T might be less sensitive to detect mild form of delirium. Considering that symptom severity of delirium tends to fluctuate during the day, serial assessment may improve the sensitivity of the test.

An earlier study of the 4AT in hospitalized elderly patients revealed that its specificity is compromised in demented population. ${ }^{17}$ Even though baseline cognitive function was not evaluated in the present study, it seems that cognitive impairment that is not caused by delirium, for example, dementia or organic brain syndrome, could contribute to false positive results. The finding is not surprising because it is generally known that clinical expertise is required to discriminate delirium from behavioral and psychological changes due to impaired cognitive function. Besides, such over-detection should be considered acceptable for screening of a serious condition such as delirium.

In Thailand, a heavy workload due to the small number of health care personnel has long been a key public health problem. Concerning delirium, this might be an important factor leading to underrecognition of the syndrome due to lack of time. In the present study, the 4AT-T was administered by attending nurses without prior specific training provided and demonstrated its brevity and ease of use. Moreover, in the medical wards where each nurse has to provide care for up to eight patients with complex conditions, the 4AT-T was still compatible with routine work confirmed by feedback from the participating nurses. From all the aforementioned findings, we propose that the $4 \mathrm{AT}-\mathrm{T}$ could be a practical delirium-screening tool for routine use in similar clinical scenarios in Thailand.

However, the 4AT-T also has some limitations. First, the test is not suitable for patients having difficulty in communication because such conditions could interfere with scoring of the AMT4 and the attention items. Second, according to the scoring system of the 4AT, solely the presence of changes in alertness or a fluctuating course of the mental status would already generate a score of 4 points, which is the cut-off score for possible delirium. Therefore, any errors in scoring of these two items, probably due to insufficient information from reliable informants or lack of evaluators' experience in assessing the alertness level, could lead to a misclassification of the condition. Moreover, considering that this study included only patients with general medical illnesses, further validation studies are required for application of the test to patients with other specific conditions such as those in postoperative or critical care settings.

\section{Conclusion}

The 4AT-T is a reliable and valid delirium-screening instrument for hospitalized elderly patients with acute medical illnesses. The tool appears practical even in high-workload settings without a need for specific training. Further validation studies in patients with other specific clinical conditions are required. 


\section{Acknowledgments}

This study was supported by a grant from the Faculty of Medicine, Ramathibodi Hospital, Mahidol University, Bangkok, Thailand. The authors are grateful to Thanita Hiranyatheb for her assistance in the revision of the manuscript.

\section{Disclosure}

The authors report no conflicts of interest in this work.

\section{References}

1. American Psychiatric Association. Diagnostic and Statistical Manual of Mental Disorders. 5th ed. Washington DC: American Psychiatric Association; 2013.

2. Inouye SK, Westendorp RG, Saczynski JS. Delirium in elderly people. Lancet. 2014;383:911-922.

3. Inouye SK. Delirium in older persons. $N$ Engl J Med. 2006;354: 1157-1165.

4. Siddiqi N, House AO, Holmes JD. Occurrence and outcome of delirium in medical in-patients: a systematic literature review. Age Ageing. 2006; 35:350-364.

5. Fong TG, Tulebaev SR, Inouye SK. Delirium in elderly adults: diagnosis, prevention and treatment. Nat Rev Neurol. 2009;5:210-220.

6. Irwin SA, Pirrello RD, Hirst JM, Buckholz GT, Ferris FD. Clarifying delirium management: practical, evidenced-based, expert recommendations for clinical practice. J Palliat Med. 2013;16:423-435.

7. Cole MG, McCusker J, Bellavance F, et al. Systematic detection and multidisciplinary care of delirium in older medical inpatients: a randomized trial. CMAJ. 2002;167:753-759.

8. Cole MG, Ciampi A, Belzile E, Zhong L. Persistent delirium in older hospital patients: a systematic review of frequency and prognosis. Age Ageing. 2009;38:19-26.

9. Kiely DK, Marcantonio ER, Inouye SK, et al. Persistent delirium predicts greater mortality. J Am Geriatr Soc. 2009;57:55-61.

10. Canadian Coalition for Seniors' Mental Health. National Guidelines for Seniors' Mental Health: The Assessment and Treatment of Delirium. Toronto: Canadian Coalition for Seniors' Mental Health; 2006.

11. National Clinical Guideline Centre for Acute and Chronic Conditions. NICE Clinical Guideline 103; Delirium: Diagnosis, Prevention and Management. London: National Institute for Health and Clinical Excellence; 2010.

12. American Psychiatric Association. Practice guideline for the treatment of patients with delirium. Am J Psychiatry. 1999;156:1-20.

13. Maldonado JR. Delirium in the acute care setting: characteristics, diagnosis and treatment. Crit Care Clin. 2008;24:657-722, vii.

14. Wong CL, Holroyd-Leduc J, Simel DL, Straus SE. Does this patient have delirium?: value of bedside instruments. JAMA. 2010;304: $779-786$.
15. Siddiqi N, Stockdale R, Britton AM, Holmes J. Interventions for preventing delirium in hospitalised patients. Cochrane Database Syst Rev. 2007;(2):CD005563.

16. Wei LA, Fearing MA, Sternberg EJ, Inouye SK. The Confusion Assessment Method: a systematic review of current usage. J Am Geriatr Soc. 2008;56:823-830.

17. Bellelli G, Morandi A, Davis DH, et al. Validation of the 4AT, a new instrument for rapid delirium screening: a study in 234 hospitalised older people. Age Ageing. 2014;43:496-502.

18. Lees R, Corbet S, Johnston C, Moffitt E, Shaw G, Quinn TJ. Test accuracy of short screening tests for diagnosis of delirium or cognitive impairment in an acute stroke unit setting. Stroke. 2013;44:3078-3083.

19. Praditsuwan R, Limmathuroskul D, Assanasen J, et al. Prevalence and incidence of delirium in Thai older patients: a study at general medical wards in Siriraj Hospital. J Med Assoc Thai. 2012;95 Suppl 2: S245-S250.

20. Limpawattana P, Sutra S, Thavornpitak Y, Sawanyawisuth K, Chindaprasirt J, Mairieng P. Delirium in hospitalized elderly patients of Thailand; is the figure underrecognized? J Med Assoc Thai. 2012;95 Suppl 7:S224-S228.

21. Yue P, Wang L, Liu C, Wu Y. A qualitative study on experience of nurses caring for patients with delirium in ICUs in China: Barriers, burdens and decision making dilemmas. Int J Nurs Sci. 2015;2:2-8.

22. Nantsupawat A, Srisuphan W, Kunaviktikul W, Wichaikhum OA, Aungsuroch Y, Aiken LH. Impact of nurse work environment and staffing on hospital nurse and quality of care in Thailand. J Nurs Scholarsh. 2011;43:426-432.

23. Noel-Storr AH, McCleery JM, Richard E, et al. Reporting standards for studies of diagnostic test accuracy in dementia: The STARDdem Initiative. Neurology. 2014;83:364-373.

24. Chester JG, Beth Harrington M, Rudolph JL, Group VADW. Serial administration of a modified Richmond Agitation and Sedation Scale for delirium screening. J Hosp Med. 2012;7:450-453.

25. Schofield I, Stott DJ, Tolson D, McFadyen A, Monaghan J, Nelson D. Screening for cognitive impairment in older people attending accident and emergency using the 4-item Abbreviated Mental Test. Eur JEmerg Med. 2010;17:340-342.

26. Katzman R, Brown T, Fuld P, Peck A, Schechter R, Schimmel H. Validation of a short orientation-memory-concentration test of cognitive impairment. Am J Psychiatry. 1983;140:734-739.

27. Zartrungpak SPR, Jennawasin S, Saipanish P. Validity of Thai Delirium Rating Scale 6 items version. Siriraj Med J. 2001;53:672-677.

28. Charlson ME, Pompei P, Ales KL, MacKenzie CR. A new method of classifying prognostic comorbidity in longitudinal studies: development and validation. J Chronic Dis. 1987;40:373-383.

29. American Psychiatric Association. Diagnostic and Statistical Manual of Mental Disorders. 4th ed. Washington DC: American Psychiatric Association; 2000.

30. Streiner DL, Cairney J. What's under the ROC? An introduction to receiver operating characteristics curves. Can J Psychiatry. 2007;52: $121-128$. 


\section{Supplementary materials}

Table SI Item level values and item-total correlation of 4AT-T

\begin{tabular}{llll}
\hline Items & Mean & SD & $\begin{array}{l}\text { Corrected item-total } \\
\text { correlation }\end{array}$ \\
\hline Alertness & 0.454 & 1.27 & 0.549 \\
AMT4 & 0.845 & 0.93 & 0.607 \\
Attention & 0.845 & 0.68 & 0.680 \\
Acute change/fluctuation & 1.113 & 1.80 & 0.649 \\
\hline
\end{tabular}

Abbreviations: 4AT-T, Thai version of the 4 'A's Test; SD, standard deviation; AMT4, 4-item Abbreviated Mental Test.

Table S2 The performance of the 4AT-T at various cut-off values in diagnosing delirium

\begin{tabular}{lllll}
\hline Criterion & Sensitivity (\%) & Specificity (\%) & LR+ \\
\hline$\geq 0$ & 100.00 & 0.00 & 1.00 & LR- \\
$>0$ & 100.00 & 39.73 & 1.66 & 2.69 \\
$>1$ & 95.83 & 64.38 & 3.52 & 0.00 \\
$>2$ & 91.67 & 73.97 & $\mathbf{6 . 0 8}$ & 0.065 \\
$>3$ & 83.33 & 86.30 & 6.42 & 0.11 \\
$>4$ & 79.17 & 87.67 & 7.22 & 0.19 \\
$>5$ & 79.17 & 89.04 & 10.95 & 0.24 \\
$>6$ & 75.00 & 93.15 & 11.15 & 0.23 \\
$>7$ & 45.83 & 95.89 & 12.17 \\
$>8$ & 33.33 & 97.26 & 24.33 & 0.27 \\
$>9$ & 33.33 & 98.63 & 21.29 \\
$>11$ & 29.17 & 98.63 & 0.56 \\
\hline 12 & 0.00 & 100.00 & 0.69
\end{tabular}

Note: Bold values represent maximal Youden's index $J=0.70$.

Abbreviations: 4AT-T, Thai version of the 4 'A's Test; LR+, positive likelihood ratio; LR-, negative likelihood ratio.

\section{Publish your work in this journal}

Neuropsychiatric Disease and Treatment is an international, peerreviewed journal of clinical therapeutics and pharmacology focusing on concise rapid reporting of clinical or pre-clinical studies on a range of neuropsychiatric and neurological disorders. This journal is indexed on PubMed Central, the 'PsycINFO' database and CAS, and is the official journal of The International Neuropsychiatric Association (INA). The manuscript management system is completely online and includes a very quick and fair peer-review system, which is all easy to use. Visit http://www.dovepress.com/testimonials.php to read real quotes from published authors. 